ALEPH 99-051

CONF 99-027

EPS-HEP99\# 6-417

28 June 1999

\title{
PRELIMINARY
}

\section{Search for Extra Spatial Dimensions and TeV Scale Quantum Gravity at LEP}

\author{
The ALEPH Collaboration
}

\begin{abstract}
A search for extra spatial dimensions in the context of $\mathrm{TeV}$ scale quantum gravity has been performed with the ALEPH detector at LEP. At LEP-2, a contribution to the production of events with single photons plus missing energy from the process $e^{+} e^{-} \rightarrow \gamma \mathrm{G}$ (with $\mathrm{G}$ a massive graviton) has been looked for, but no significant signal has been found. Lower limits at the $95 \%$ confidence level on the mass scale $M_{D}$, at which gravity becomes strong, are placed in the range $0.99 \mathrm{TeV}$ to $0.49 \mathrm{TeV}$ for 2 to 6 extra spatial dimensions, respectively. The corresponding upper limits on the size of the extra spatial dimensions are $0.49 \mathrm{~mm}$ to $69 \mathrm{fm}$. With LEP-1 data, a search for scalar graviton $\left(\mathrm{G}_{0}\right)$ production in the $\mathrm{Z}$ decay channel $\mathrm{Z} \rightarrow \mathrm{f} \bar{f} \mathrm{G}_{0}$ has been performed, but no signal has been found, leading to limits on $M_{D}$ in the range 0.35 to $0.12 \mathrm{TeV}$. Additional searches have been made at LEP-2 for modifications due to virtual graviton exchange to the $e^{+} e^{-} \rightarrow \gamma \gamma$ and $e^{+} e^{-} \rightarrow \mathrm{f} \bar{f}$ differential cross sections. No significant deviations from Standard Model expectations have been found. Lower limits on the ultraviolet cutoff parameters $\Lambda_{T}^{+}$and $\Lambda_{T}^{-}$are established as $1.12 \mathrm{TeV}$ and $0.84 \mathrm{TeV}$, respectively, at the $95 \%$ confidence level.
\end{abstract}

ALEPH contribution to the Summer, 1999 conferences

Contacts:

alan.litke@cern.ch

marcello.maggi@cern.ch

gary.taylor@cern.ch

ian.tomalin@cern.ch 


\section{Introduction}

Recently, Arkani-Hamed, Dimopoulos and Dvali (ADD) [1] have proposed a solution to the hierarchy problem which does not rely on low energy supersymmetry or technicolor. In this new framework, gravity is characterized by a fundamental mass scale $M_{D}$ of order $\sim \mathrm{TeV}$, and space consists of $\delta$ extra dimensions of size $R$, with

$$
G_{N}^{-1}=8 \pi R^{\delta} M_{D}^{2+\delta}
$$

where $G_{N}$ is the Newtonian gravitational constant.

In this $3+\delta$ dimensional space, the graviton propagates as a massless, spin-2 particle. However, in projection onto our normal world of three spatial dimensions, it appears as a spectrum of massive Kaluza-Klein excitations, with spins zero, one and two. The spin-2 gravitons $(\mathrm{G})$ couple in the usual way to the stress-energy tensor of the Standard Model (SM) fields. They have very weak jnteraction with matter (of gravitational strength) and very long lifetime. The spin-1 gravitons decouple from ordinary matter, and the scalar gravitons $\left(G_{0}\right)$ couple to SM fermions (gauge bosons) with a strength proportional to the particle mass (mass squared).

This theory has some striking experimental signatures, including the breakdown of the Newtonian gravitational law $V(r) \sim 1 / r$ for distances $r<R$. More specifically, the gravitational 送 force increases rapidly at distances $r$ less than $R$ with a $1 / r^{2+\delta}$ dependence, becoming strong at $r \sim 1 / M_{D}$. Present measurements exclude $\delta=1$, but $\delta=2$, with $R \sim 100 \mu \mathrm{m}$, cannot yet be ruled out by direct laboratory measurement ${ }^{1}$.

This note reports on four measurements accessible to ALEPH which probe the experimental consequences of extra spatial dimensions and $\mathrm{TeV}$ scale quantum gravity:

1. the search for direct spin-2 graviton $(\mathrm{G})$ production in the reaction $e^{+} e^{-} \rightarrow \gamma \mathrm{G}$, in the center of mass energy range $161 \leq \sqrt{s} \leq 189 \mathrm{GeV}^{2}$

2. the search for direct scalar graviton production in the $Z$ decay channel $Z \rightarrow f \bar{f} G_{0}$, where $f \bar{f}$ represents a fermion-anti-fermion pair;

3. the search for a deviation from the SM differential cross section for $e^{+} e^{-} \rightarrow \gamma \gamma$ due to virtual graviton exchange, in the energy range $161 \leq \sqrt{s} \leq 189 \mathrm{GeV}$; and

4. the search for a deviation from the SM differential cross sections for $e^{+} e^{-} \rightarrow \mathrm{f} \bar{f}$ due to virtual graviton exchange, in the energy range $130 \leq \sqrt{s} \leq 189 \mathrm{GeV}$.

The results presented below are based on ALEPH measurements either already published or submitted to recent conferences. The measurements (1) and (2), connected with a direct search for graviton production, will be parametrized in terms of the gravitational mass scale $M_{D}$, and equivalently (via equation 1 ), with the size $R$ of the extra spatial dimensions. Measurements (3) and (4), related to virtual graviton exchange, will be characterized in terms of an ultraviolet cutoff energy $\Lambda_{T}[2,3,4]$. In the absence of a full theory of quantum gravity, this cutoff energy cannot be precisely calculated in terms of the mass scale $M_{D}$, but the ratio $\Lambda_{T} / M_{D}$ is expected to be of order unity.

\footnotetext{
${ }^{1}$ Astrophysical measurements have been used to set lower limits on $M_{D}$ at the multi-TeV level for $\delta=2$. See, for example, S. Cullen and M. Perelstein, "SN1987A Constraints on Large Compact Dimensions", SLAC-PUB-8084, SU-ITP-99/15 (March, 1999); hep-ph/9903422.

${ }^{2}$ In this reaction, scalar graviton production is negligible as its coupling is proportional to the electron mass; only spin-2 graviton production plays a significant role.
} 


\section{$2 \quad e^{+} e^{-} \rightarrow \gamma \mathrm{G}$}

In the ADD scenario, a spectrum of massive gravitons can be produced in association with a photon in $e^{+} e^{-}$annihilation. The differential cross section for this process depends on both $M_{D}$ and $\delta$ and is given by [4]:

$$
\frac{d^{2} \sigma}{d x d \cos \theta}=\frac{\alpha}{32 s} \frac{\pi^{\delta / 2}}{\Gamma(\delta / 2)}\left(\frac{\sqrt{s}}{M_{D}}\right)^{\delta+2} f(x, \cos \theta)
$$

with:

$$
f(x, \cos \theta)=\frac{2(1-x)^{\frac{\delta}{2}-1}}{x\left(1-\cos ^{2} \theta\right)}\left[(2-x)^{2}\left(1-x+x^{2}\right)-3 x^{2}(1-x) \cos ^{2} \theta-x^{4} \cos ^{4} \theta\right]
$$

where $x$ is the ratio of the photon energy to the beam energy, and $\theta$ is the angle between the photon and beam directions.

As noted in section 1 , the gravitons $\mathrm{G}$ are characterized by very weak interaction with matter and very long lifetime $\left(c \tau_{\mathrm{G}} \geq 1.5 \times 10^{16} \mathrm{~cm}\right.$ for $M_{\mathrm{G}} \leq 200 \mathrm{GeV}$, the kinematic limit for production at LEP-2). With these properties, on the scale of the ALEPH detector, G is a non-interacting stable particle. Its production can be detected through the observation of a single photon and missing energy $\not E$.

The reaction $e^{+} e^{-} \rightarrow \gamma \not E$ is a well-studied process in ALEPH at LEP-2 [5, 6, 7]. Summary plots for all the data collected in the center of mass energy range 161-189 GeV are shown in figure 1. These data were taken at center of mass energies of $161,172,182.7$, and $188.6 \mathrm{GeV}$ with corresponding integrated luminosities of $11.1,10.6,58.5$, and $173.6 \mathrm{pb}^{-1}$. The event selection efficiency is $74 \%$ for photons inside the acceptance $|\cos \theta|<0.95$ and $p_{\perp}<0.0375 \sqrt{s}$. The total number of events selected is 759, with an expected value from SM processes of 763 .

Figure 1a displays the invariant mass $\left(M_{\text {miss }}\right)$ distribution of the system recoiling against the photon candidate, for the combined data sample. This distribution is dominated by radiative returns to the $\mathrm{Z}$, where the $\mathrm{Z}$ decays to $\nu \bar{\nu}$. The polar angle $(\theta)$ distribution for the photon candidates is shown in figure $1 \mathrm{~b}$. There is seen to be good agreement between the data and the SM expectation.

The search for $e^{+} e^{-} \rightarrow \gamma \mathrm{G}$ is based on a binned maximum likelihood fit to the two-dimensional distribution in $M_{\text {miss }}$ and $\cos \theta$ for the photon candidates. The fit is performed under the hypothesis that the data contains a mixture of signal and background, with the differential cross section for the signal given in equations 2 and 3. Initial state radiation is taken into account. The 1.8\% systematic uncertainty on the background level [7] is included in the likelihood function.

The fit parameter $\epsilon$ is taken to be $\epsilon=\left(1 / M_{D}\right)^{\delta+2}$ for $\delta$ extra dimensions; this provides a likelihood distribution $\mathcal{L}(\epsilon)$ which is well described by a Gaussian function (truncated for $\epsilon<0$ as the signal cross section, which is proportional to $\epsilon$, must be $\geq 0$ ). The fit results are displayed in Table 1.

As no statistically significant signal is observed, $95 \%$ confidence level lower limits $M_{D 95}$ are placed on the fundamental mass scale $M_{D}$ as a function of the number of extra dimensions $\delta$. $M_{D 95}$ is defined by $M_{D 95}=\left(\epsilon_{95}\right)^{-1 /(\delta+2)}$ with:

$$
\int_{0}^{\epsilon 95} \mathcal{L}\left(\epsilon^{\prime}\right) d \epsilon^{\prime}=0.95 \int_{0}^{\infty} \mathcal{L}\left(\epsilon^{\prime}\right) d \epsilon^{\prime} .
$$

These limits are shown in Table 1 along with the corresponding upper limits $R_{95}$ on the size $R$ of the extra spatial dimensions derived from equation 1. 

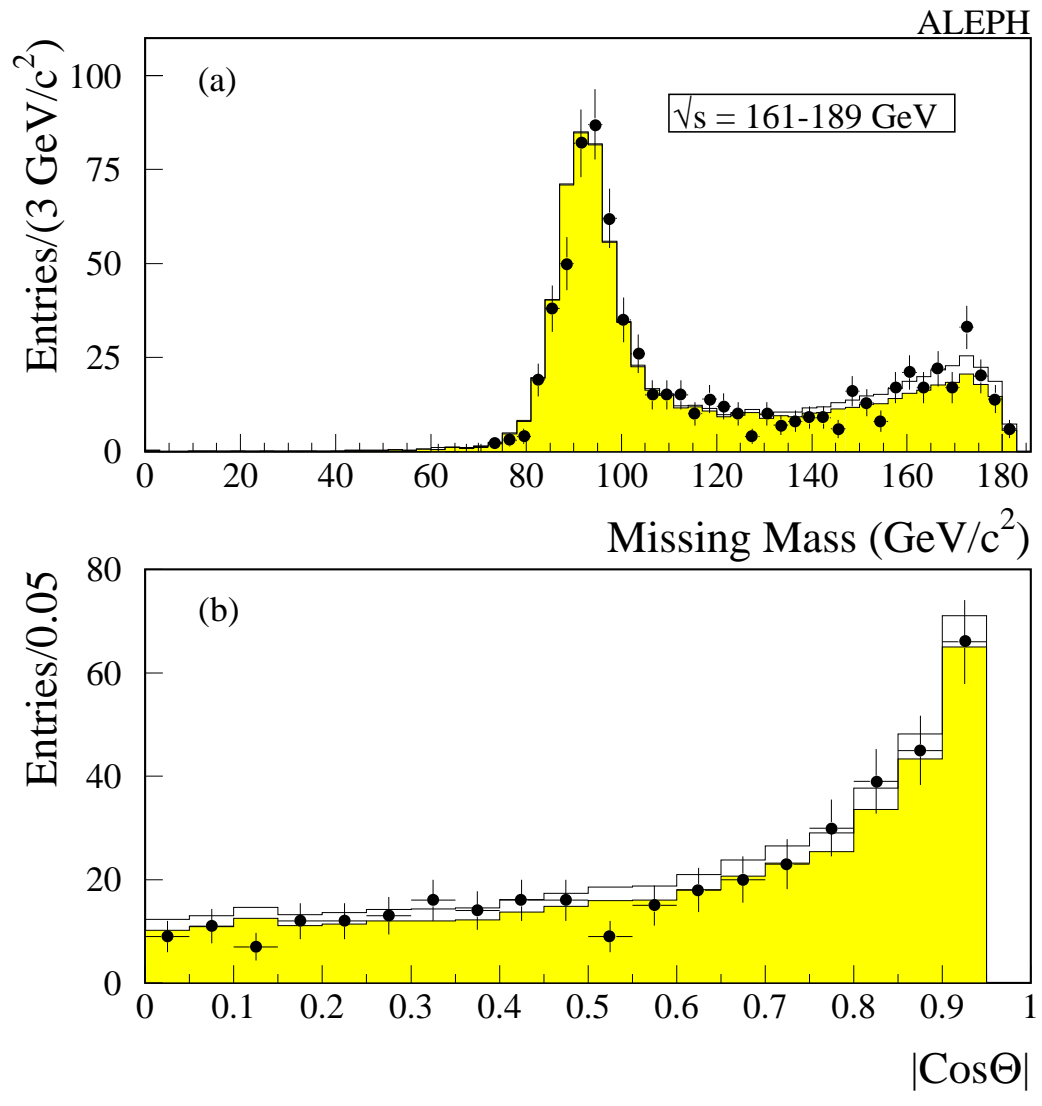

Figure 1: (a) The invariant mass $\left(M_{\text {miss }}\right)$ distribution of the system recoiling against the candidate photon. (b) The polar angle $(\theta)$ distribution for the candidate photon. These plots show the data (points with error bars) for the combined data sample $(\sqrt{s}=161-189 \mathrm{GeV})$, the absolutely normalized SM expectation (shaded histogram), and the expected $\gamma \mathrm{G}$ signal (open histogram) for two extra dimensions, with $M_{D}$ set to the $95 \%$ confidence level lower limit of $0.99 \mathrm{TeV}$.

\begin{tabular}{|c|c|c|c|}
\hline$\delta$ & $\left(1 / M_{D}\right)^{\delta+2}$ & $M_{D 95}(\mathrm{TeV})$ & $R_{95}(\mathrm{~cm})$ \\
\hline \hline 2 & $0.46 \pm 0.34 \mathrm{TeV}^{-4}$ & 0.99 & $4.9 \times 10^{-2}$ \\
3 & $1.57 \pm 1.08 \mathrm{TeV}^{-5}$ & 0.78 & $5.4 \times 10^{-7}$ \\
4 & $6.33 \pm 4.24 \mathrm{TeV}^{-6}$ & 0.65 & $1.9 \times 10^{-9}$ \\
5 & $29.1 \pm 19.3 \mathrm{TeV}^{-7}$ & 0.55 & $6.4 \times 10^{-11}$ \\
6 & $150 . \pm 99 . \mathrm{TeV}^{-8}$ & 0.49 & $6.9 \times 10^{-12}$ \\
\hline
\end{tabular}

Table 1: Fitted values for $\left(1 / M_{D}\right)^{\delta+2}$, the lower limits $M_{D 95}$ on the mass scale $M_{D}$, and upper limits $R_{95}$ on the size of the extra dimensions $R$, for $\delta$ extra dimensions. All the limits are at $95 \%$ confidence level. 


\begin{tabular}{|c|c|c|c|}
\hline$\delta$ & $B R\left(\right.$ with $M_{D}$ in TeV) & $\langle$ efficiency $\rangle$ & $M_{D 95}(\mathrm{TeV})$ \\
\hline \hline 2 & $4.0 \times 10^{-8} / M_{D}^{4}$ & 0.26 & 0.35 \\
3 & $1.3 \times 10^{-9} / M_{D}^{5}$ & 0.32 & 0.22 \\
4 & $4.5 \times 10^{-11} / M_{D}^{6}$ & 0.34 & 0.17 \\
5 & $1.9 \times 10^{-12} / M_{D}^{7}$ & 0.34 & 0.14 \\
6 & $7.5 \times 10^{-14} / M_{D}^{8}$ & 0.32 & 0.12 \\
\hline
\end{tabular}

Table 2: The branching ratios $B R \equiv \Gamma\left(\mathrm{Z} \rightarrow \mathrm{f} \overline{\mathrm{f}} \mathrm{G}_{0}\right) / \Gamma(\mathrm{Z} \rightarrow \mathrm{f} \overline{\mathrm{f}})$ tabulated from equation 5 , the average selection efficiencies $\langle$ efficiency $\rangle$, and the corresponding $95 \%$ confidence level lower limits $M_{D 95}$ on $M_{D}$ for $\delta$ extra dimensions.

\section{$3 \quad \mathrm{Z} \rightarrow \mathrm{f} \overline{\mathrm{f}} \mathrm{G}_{0}$}

In this section a search for scalar gravitons, based on the coupling of the $G_{0}$ to the $Z$, is described (as noted in section 1, this coupling is much stronger than that of $G$ to the electron). The search channel is the decay $\mathrm{Z} \rightarrow \mathrm{Z}^{*} \mathrm{G}_{0}$, followed by $\mathrm{Z}^{*} \rightarrow \mathrm{f} \overline{\mathrm{f}}$. The expected $\mathrm{Z}$ branching ratio is proportional to $\left(M_{\mathrm{Z}} / M_{D}\right)^{\delta+2}$ and is given by [8]:

$$
B R \equiv \frac{\Gamma\left(\mathrm{Z} \rightarrow \mathrm{f} \overline{\mathrm{f}} \mathrm{G}_{0}\right)}{\Gamma(\mathrm{Z} \rightarrow \mathrm{f} \overline{\mathrm{f}})}=\frac{1}{4 \pi} \frac{1}{3(\delta+2)}\left(\frac{M_{\mathrm{Z}}}{M_{D}}\right)^{\delta+2} I
$$

where the integral $I$ is defined as:

$$
I=\frac{\pi^{(\delta-2) / 2}}{\Gamma(\delta / 2)} \int_{0}^{1} \int_{0}^{(1-\sqrt{x})^{2}} d x d y \frac{y^{(\delta-2) / 2}(12 x+A) \sqrt{A}}{6(1-x)^{2}}
$$

with $A=(1-x-y)^{2}-4 x y$. Numerical results for $B R$ are shown in Table 2 .

The signature for this process is the presence of a virtual $\mathrm{Z}$ decay to $\mathrm{f} \overline{\mathrm{f}}$ accompanied by missing energy carried away by a scalar particle. This topolgy is identical to that already looked for in ALEPH in the search for invisible decays of the Higgs boson $h$ in the process $\mathrm{Z} \rightarrow \mathrm{Z}^{*} \mathrm{~h}$ with $\mathrm{Z}^{*} \rightarrow \mathrm{f} \overline{\mathrm{f}}[9]$.

The following results are based on the search for acoplanar jet pairs in $4 \times 10^{6}$ hadronic Z decays, as described in [9]. This search topology provides the best expected limits. The average efficiency for signal events is shown in Table 2 as a function of $\delta$. It is calculated by folding together the expected $\mathrm{G}_{0}$ mass spectrum [8] with the efficiency curve given in [9] as a function of $M_{h}$.

No candidate events are found, with an expected background of 1.4 events. The $95 \%$ confidence level lower limits $M_{D 95}$ on $M_{D}$, taking the number of signal events to be less than three, are tabulated in Table 2. When these measurements and limits on $M_{D}$ are combined with those from section 2, the results displayed in Table 1 are left unchanged.

\section{$4 e^{+} e^{-} \rightarrow \gamma \gamma$}

In the $\mathrm{ADD}$ framework, the $e^{+} e^{-} \rightarrow \gamma \gamma \mathrm{SM}$ cross section is modified due to additional amplitudes in which a spectrum of virtual gravitons are exchanged in the s-channel. The modified cross section is given by [4]: 


$$
\frac{d \sigma}{d \Omega}=\frac{1}{s}\left[\alpha^{2} \frac{\left(1+\cos ^{2} \theta\right)}{\left(1-\cos ^{2} \theta\right)}+\frac{\alpha s^{2}}{4}\left(\frac{1}{\Lambda_{T}}\right)^{4}\left(1+\cos ^{2} \theta\right)+\frac{s^{4}}{64}\left(\frac{1}{\Lambda_{T}}\right)^{8}\left(1-\cos ^{4} \theta\right)\right]
$$

where $s$ is the square of the center of mass energy and $\theta$ is the photon production angle. The first term is the SM cross section; the two additional terms, due to virtual graviton exchange, are expressed in terms of the ultraviolet cutoff energy $\Lambda_{T}$ introduced in section 1 .

A slightly generalized version of equation 6 will be used in quoting the experimental results. This modified version allows for the possibility of a complex phase in the interference term between the SM and graviton exchange amplitudes. For simplicity, only the phase factors +1 and -1 will be considered, with the corresponding cutoff parameters $\Lambda_{T}^{+}$and $\Lambda_{T}^{-}$. The modified cross-section is:

$$
\frac{d \sigma}{d \Omega}=\frac{1}{s}\left[\alpha^{2} \frac{\left(1+\cos ^{2} \theta\right)}{\left(1-\cos ^{2} \theta\right)} \pm \frac{\alpha s^{2}}{4}\left(\frac{1}{\Lambda_{T}^{ \pm}}\right)^{4}\left(1+\cos ^{2} \theta\right)+\frac{s^{4}}{64}\left(\frac{1}{\Lambda_{T}^{ \pm}}\right)^{8}\left(1-\cos ^{4} \theta\right)\right]
$$

The reaction $e^{+} e^{-} \rightarrow \gamma \gamma$ has also been well studied in ALEPH at LEP-2 [5, 6, 7]. Indeed, the search for effects due to virtual graviton exchange follows closely the search for effects due to contact interactions or the exchange of an excited electron.

The photon polar angle distribution, using the combined data sample for center of mass energies 161-189 GeV, is shown in figure 2 along with the SM expectation. This distribution is plotted in terms of the production angle $\theta^{*}$ in the two-photon rest-frame. The total number of selected events is 2022, which can be compared to the expected number of 2112 .

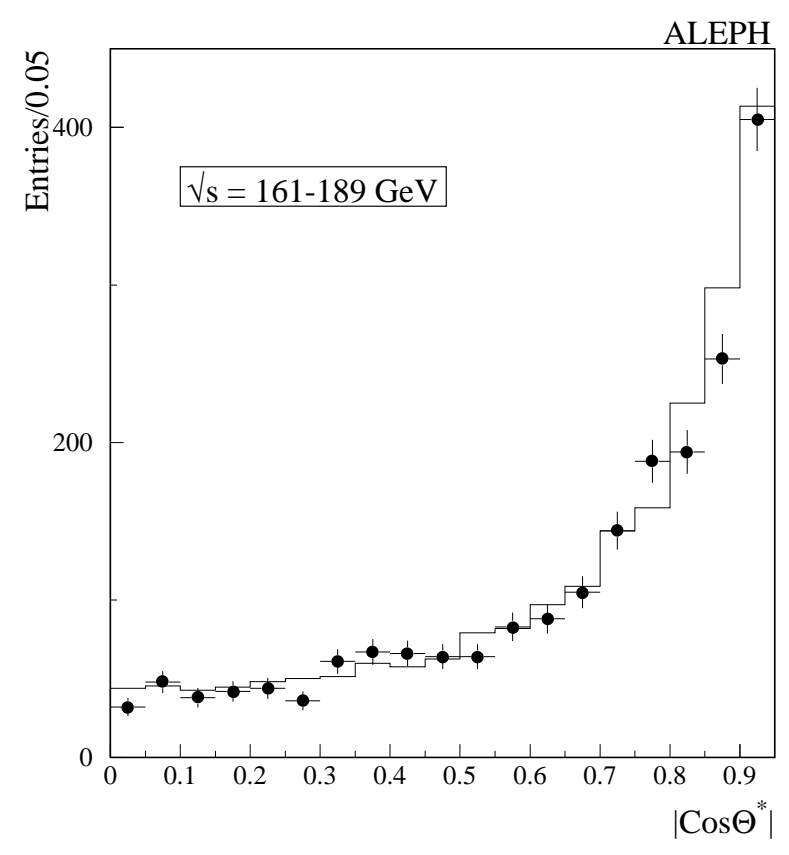

Figure 2: The production angle $\left(\theta^{*}\right)$ distribution for $e^{+} e^{-} \rightarrow \gamma \gamma$, with $\left|\cos \theta^{*}\right|$ calculated in the twophoton rest-frame. The data (points with error bars) are the combined sample for $\sqrt{s}=161-189$ $\mathrm{GeV}$; only statistical errors are shown. The prediction from the SM (histogram) is absolutely normalized and includes a small contribution from Bhabha background. 
With the data displayed in figure 2, a binned maximum likelihood fit is performed for the parameter $\epsilon=\left(1 / \Lambda_{T}\right)^{4}$. The fit is based on the differential cross section, equation 6 , corrected for detector efficiency and higher order corrections (assumed to be the same as for the SM alone). The systematic uncertainty of $2.2 \%$ [7] on the measurement is taken into account in the likelihood function. The fit gives:

$$
\epsilon=\left(1 / \Lambda_{T}\right)^{4}=-0.97 \pm 1.09 \mathrm{TeV}^{-4}
$$

with the likelihood distribution $\mathcal{L}(\epsilon)$ well-described by a Gaussian distribution.

As there is no statistically significant evidence for a non-zero value of $\epsilon=\left(1 / \Lambda_{T}\right)^{4}$, the results will be quoted in terms of lower limits on $\Lambda_{T}^{+}$and $\Lambda_{T}^{-}$, with $\left(1 / \Lambda_{T}^{ \pm}\right)^{4}= \pm\left(1 / \Lambda_{T}\right)^{4}$. As $\left(1 / \Lambda_{T}^{ \pm}\right)^{4}$ must be positive, the $95 \%$ confidence level lower limit $\Lambda_{T 95}^{+}$on $\Lambda_{T}^{+}$is defined as $\Lambda_{T 95}^{+}=\left(\epsilon_{95}^{+}\right)^{-1 / 4}$ where

$$
\int_{0}^{\epsilon_{95}^{+}} \mathcal{L}\left(\epsilon^{\prime}\right) d \epsilon^{\prime}=0.95 \int_{0}^{\infty} \mathcal{L}\left(\epsilon^{\prime}\right) d \epsilon^{\prime}
$$

Likewise, the $95 \%$ confidence level lower limit $\Lambda_{T 95}^{-}$on $\Lambda_{T}^{-}$is defined as $\Lambda_{T 95}^{-}=\left(-\epsilon_{95}^{-}\right)^{-1 / 4}$ where

$$
\int_{\epsilon_{95}^{-}}^{0} \mathcal{L}\left(\epsilon^{\prime}\right) d \epsilon^{\prime}=0.95 \int_{-\infty}^{0} \mathcal{L}\left(\epsilon^{\prime}\right) d \epsilon^{\prime} .
$$

Numerically it is found that $\Lambda_{T 95}^{+}=0.89 \mathrm{TeV}$ and $\Lambda_{T 95}^{-}=0.77 \mathrm{TeV}$. The ratio of the measured differential cross section to the SM prediction is shown in figure 3 as a function of $\left|\cos \theta^{*}\right|$. Also indicated are the predictions for this ratio in the ADD scenario with $\Lambda_{T}^{ \pm}$set to their lower limit values.

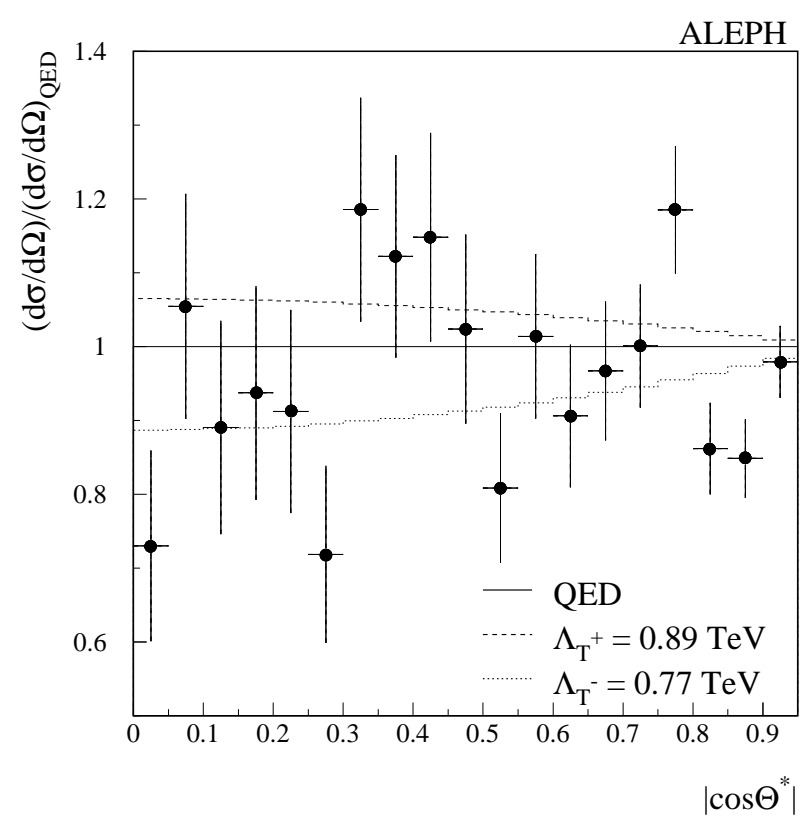

Figure 3: The ratio of the observed to the predicted differential cross section for $e^{+} e^{-} \rightarrow \gamma \gamma$ as a function of $\left|\cos \theta^{*}\right|$, where $\theta^{*}$ is the photon production angle in the two-photon rest-frame. The dashed and dotted lines correspond to the cross section ratios which include the effects from virtual graviton exchange; $\Lambda_{T}^{+}\left(\Lambda_{T}^{-}\right)$is set equal to its $95 \%$ C.L. lower limit of $0.89(0.77) \mathrm{TeV}$. 


\section{$5 \quad e^{+} e^{-} \rightarrow \mathrm{f} \overline{\mathrm{f}}$}

\subsection{Born Cross Section:}

As in the case of $e^{+} e^{-} \rightarrow \gamma \gamma$ described in section 4 , the $e^{+} e^{-} \rightarrow \mathrm{f} \overline{\mathrm{f}} \mathrm{SM}$ differential cross section is modified in the ADD framework due to additional s-channel graviton exchange amplitudes. The modified cross section is [4]:

$$
\begin{aligned}
& \frac{d \sigma}{d t}\left(\mathrm{e}^{+} \mathrm{e}^{-} \rightarrow \mathrm{f} \overline{\mathrm{f}}\right)=\frac{d \sigma}{d t}\left(\mathrm{e}^{+} \mathrm{e}^{-} \rightarrow \mathrm{f} \overline{\mathrm{f}}\right)_{\mathrm{SM}} \\
& -\frac{N_{\mathrm{f}} \alpha \pi}{2 \Lambda_{T}^{4}}\left\{Q_{\mathrm{e}} Q_{\mathrm{f}} G_{5}(t / s)+\frac{1}{\sin ^{2} 2 \theta_{\mathrm{W}}} \frac{s}{s-M_{\mathrm{Z}}^{2}}\left[v_{\mathrm{e}} v_{\mathrm{f}} G_{5}(t / s)+a_{\mathrm{e}} a_{\mathrm{f}} G_{6}(t / s)\right]\right\} \\
& -\frac{\alpha \pi}{2 \Lambda_{T}^{4}} \delta_{\mathrm{ef}}\left\{Q_{\mathrm{e}}^{2} G_{7}(t / s)+\frac{1}{\sin ^{2} 2 \theta_{\mathrm{W}}} \frac{s}{s-M_{\mathrm{Z}}^{2}}\left[v_{\mathrm{e}}^{2}+a_{\mathrm{e}}^{2}\right] G_{8}(t / s)+\right. \\
& \left.+\frac{1}{\sin ^{2} 2 \theta_{\mathrm{W}}} \frac{s}{t-M_{\mathrm{Z}}^{2}}\left[v_{\mathrm{e}}^{2} G_{9}(t / s)+a_{\mathrm{e}}^{2} G_{10}(t / s)\right]\right\} \\
& +\frac{N_{\mathrm{f}} \pi}{32} \frac{s^{2}}{\Lambda_{T}^{8}} G_{4}(t / s)+\frac{\delta_{\mathrm{ef}} \pi}{32} \frac{s^{2}}{\Lambda_{T}^{8}} G_{11}(t / s)
\end{aligned}
$$

where $v_{\mathrm{f}}=T_{\mathrm{f}}-2 Q_{\mathrm{f}} \sin ^{2} 2 \theta_{\mathrm{W}}, a_{\mathrm{f}}=T_{\mathrm{f}}$ and $t=\left(p_{\mathrm{e}^{-}}-p_{\mathrm{f}}\right)^{2}$. The symbol $\delta_{\text {ef }}$ is equal to 1 for electron pair production $(\mathrm{f}=\mathrm{e})$ and is equal to zero otherwise. The functions $G_{i}(x)$ are given in [4]. The parameter $\Lambda_{T}$ is the ultraviolet cutoff energy introduced in section 1 .

The first term on the right-hand side of equation 10 is the SM cross section. The interference between the SM and the graviton exchange amplitudes gives rise to the terms of order $1 / \Lambda_{T}^{4}$, whereas the pure graviton exchange produces the terms of order $1 / \Lambda_{T}^{8}$. Because the graviton is spin $2, d \sigma / d \cos \theta$ is a fourth order polynomial in $\cos \theta$ (for $\mathrm{f} \neq \mathrm{e}$ ). This gives it a signature which is very different from the other kinds of new physics considered previously [10, 11]. A further feature of the theory is that for $\mathrm{f} \neq \mathrm{e}$, the total difermion cross sections are almost unchanged from the SM predictions. This arises because the interference term in equation 10 gives zero when integrated over all polar angles and the pure graviton term is suppressed by $1 / \Lambda_{T}^{8}$.

\subsection{Higher Order Corrections:}

Equation 10 is derived in the Born approximation. Following the approach used in [10], this equation can be rendered more accurate by rewriting it as follows

$$
\frac{d \sigma}{d \cos \theta}=F_{\mathrm{SM}}(s, t)\left[1+\epsilon \frac{F_{\mathrm{IF}}^{\mathrm{Born}}(s, t)}{F_{\mathrm{SM}}^{\mathrm{Born}}(s, t)}+\epsilon^{2} \frac{F_{\mathrm{G}}^{\text {Born }}(s, t)}{F_{\mathrm{SM}}^{\mathrm{Born}}(s, t)}\right]
$$

with $\epsilon=\left(1 / \Lambda_{T}\right)^{4}$. The function $F_{\mathrm{SM}}$ is the SM cross section, which is computed using BHWIDE [12] for the $\mathrm{e}^{+} \mathrm{e}^{-}$channel and ZFITTER v6.04 [13] for the other channels. The contributions to the cross section from the SM-graviton interference and from the pure graviton exchange are denoted by $\epsilon F_{\mathrm{IF}}^{\mathrm{Born}}$ and $\epsilon^{2} F_{\mathrm{G}}^{\mathrm{Born}}$, respectively. They are calculated in the improved Born approximation. The Born level formula of equation 10 is corrected for ISR according to Ref. [14]. Because no higher order calculations are available for the gravitational interactions, the ratios of these functions with the improved Born predictions for the SM cross sections are taken. This allows for a partial cancellation of higher order effects. 
As with equation 7 , a modified version of equation 11 will be used to allow for the possibility of an induced $180^{\circ}$ relative phase between the graviton and SM amplitudes:

$$
\frac{d \sigma}{d \cos \theta}=F_{\mathrm{SM}}(s, t)\left[1 \pm \epsilon_{ \pm} \frac{F_{\mathrm{IF}}^{\mathrm{Born}}(s, t)}{F_{\mathrm{SM}}^{\mathrm{Born}}(s, t)}+\epsilon_{ \pm}^{2} \frac{F_{\mathrm{G}}^{\mathrm{Born}}(s, t)}{F_{\mathrm{SM}}^{\mathrm{Born}}(s, t)}\right]
$$

with $\epsilon_{ \pm}=\left(1 / \Lambda_{T}^{ \pm}\right)^{4}$.

\subsection{Results:}

ALEPH results on difermion production at center of mass energies $130-189 \mathrm{GeV}$ are given in $[10,11]$. These include measurements of the differential cross sections $d \sigma / d(\cos \theta)$ for $\mathrm{e}^{+} \mathrm{e}^{-}, \mu^{+} \mu^{-}$ and $\tau^{+} \tau^{-}$production, the total $\mathrm{q} \overline{\mathrm{q}}$ cross section, the ratio $R_{b}$ of the $\mathrm{b} \overline{\mathrm{b}}$ to $\mathrm{q} \overline{\mathrm{q}}$ cross sections, and measurements of jet-charge forward-backward asymmetries in $b \bar{b}$-enriched and $b \bar{b}$-depleted event samples.

A maximum likelihood fit is made to these measurements using Equation 11 with $\epsilon=\left(1 / \Lambda_{T}\right)^{4}$ being the fit parameter. The likelihood function used takes into account correlated and uncorrelated experimental systematic uncertainties and also theoretical uncertainties on the SM predictions. The SM cross sections are uncertain by $1.5 \%$ for the $\mathrm{q} \overline{\mathrm{q}}$ channel and $2 \%$ for the $\mu^{+} \mu^{-}$, $\tau^{+} \tau^{-}, \mathrm{b} \overline{\mathrm{b}}$ and $\mathrm{c} \overline{\mathrm{c}}$ channels. The SM forward-backward asymmetries have a relative uncertainty of $2.5 \%$ for each q $\bar{q}$ flavour and $4 \%$ for the $\mu^{+} \mu^{-}$and $\tau^{+} \tau^{-}$channels. The Bhabha channel is a special case because of the large $t$-channel contribution. The total Bhabha cross section, which is measured in the angular range $|\cos \theta|<0.9$, is uncertain by $2.5 \%$. Uncertainty in the angular distribution of the Bhabhas is modelled by allowing for an additional $2.5 \%$ variation in SM cross section in the region $-0.9<\cos \theta<0.7$, with the SM cross section in the region $0.7<\cos \theta<0.9$ being varied in the opposite direction to leave the total cross section unchanged. The likelihood function $\mathcal{L}(\epsilon)$ is given in full in Equation 11 of Ref. [10].

Table 3 gives the fitted values ${ }^{3}$ of $\epsilon=\left(1 / \Lambda_{T}\right)^{4}$ based on the published difermion (f $\bar{f}$ ) measurements from 130-183 GeV [10], plus the preliminary measurements at $189 \mathrm{GeV}$ [11]. Also shown are the $95 \%$ confidence level lower limits $\Lambda_{T 95}^{+}$and $\Lambda_{T 95}^{-}$on $\Lambda_{T}^{+}$and $\Lambda_{T}^{-}$(defined in equations 8 and 9). To show the relative contributions of each channel, the limits obtained separately from $\mathrm{q} \overline{\mathrm{q}}$ events (cross section and jet charge asymmetry), b $\overline{\mathrm{b}}$ events ( $R_{b}$ and jet charge asymmetry) and the three dilepton differential cross sections are also given. The likelihood function for the combined f $\bar{f}$ result is nearly Gaussian in shape.

Figure 4 illustrates these results for the Bhabha channel, which dominates the limits. The high sensitivity of this channel arises from the interference of the $s$-channel graviton exchange with the $t$-channel SM photon exchange. The Bhabha channel would be even more powerful, were it not that the theoretical errors on its SM cross section are comparable to the experimental errors. If

${ }^{3}$ The central value $\epsilon^{0}$ of $\epsilon$ is defined by

$$
\int_{\epsilon^{0}}^{\infty} \mathcal{L}\left(\epsilon^{\prime}\right) d \epsilon^{\prime}=0.5 \int_{-\infty}^{\infty} \mathcal{L}\left(\epsilon^{\prime}\right) d \epsilon^{\prime}
$$

and the $\pm 1 \sigma$ (68\% confidence level) limits $\left(\epsilon^{+}\right.$and $\left.\epsilon^{-}\right)$on $\epsilon$ are defined as

$$
\int_{-\infty}^{\epsilon^{-}} \mathcal{L}\left(\epsilon^{\prime}\right) d \epsilon^{\prime}=\int_{\epsilon^{+}}^{\infty} \mathcal{L}\left(\epsilon^{\prime}\right) d \epsilon^{\prime}=0.16 \int_{-\infty}^{\infty} \mathcal{L}\left(\epsilon^{\prime}\right) d \epsilon^{\prime}
$$




\begin{tabular}{|c||c|c|c|}
\hline Channel & $\epsilon=\left(1 / \Lambda_{T}\right)^{4}\left(\mathrm{TeV}^{-4}\right)$ & $\Lambda_{T 95}^{+}(\mathrm{TeV})$ & $\Lambda_{T 95}^{-}(\mathrm{TeV})$ \\
\hline \hline $\mathrm{q} \overline{\mathrm{q}}$ & $1.4_{-3.2}^{+3.2}$ & 0.61 & 0.66 \\
$\mathrm{~b} \overline{\mathrm{b}}$ & $3.1_{-6.1}^{+4.9}$ & 0.55 & 0.55 \\
$\mathrm{e}^{+} \mathrm{e}^{-}$ & $-1.23_{-0.70}^{+0.73}$ & 1.03 & 0.80 \\
$\mu^{+} \mu^{-}$ & $-1.2_{-2.8}^{+2.8}$ & 0.68 & 0.63 \\
$\tau^{+} \tau^{-}$ & $0.6_{-4.7}^{+4.5}$ & 0.59 & 0.57 \\
\hline $\mathrm{f} \overline{\mathrm{f}}$ & $-1.12_{-0.65}^{+0.69}$ & 1.04 & 0.82 \\
\hline
\end{tabular}

Table 3: Fitted value of $\epsilon=\left(1 / \Lambda_{T}\right)^{4}$ and the $95 \%$ confidence level lower limits $\Lambda_{T 95}^{+}$and $\Lambda_{T 95}^{-}$ obtained using the various difermion channels from $130-189 \mathrm{GeV}$. The results given for $\mathrm{f} \overline{\mathrm{f}}$ represent the combination of all channels.

the theoretical errors were zero, then the uncertainty on $\left(1 / \Lambda_{T}\right)^{4}$ from the Bhabha channel would decrease from \pm 0.72 to $\pm 0.46 \mathrm{TeV}^{-4}$.

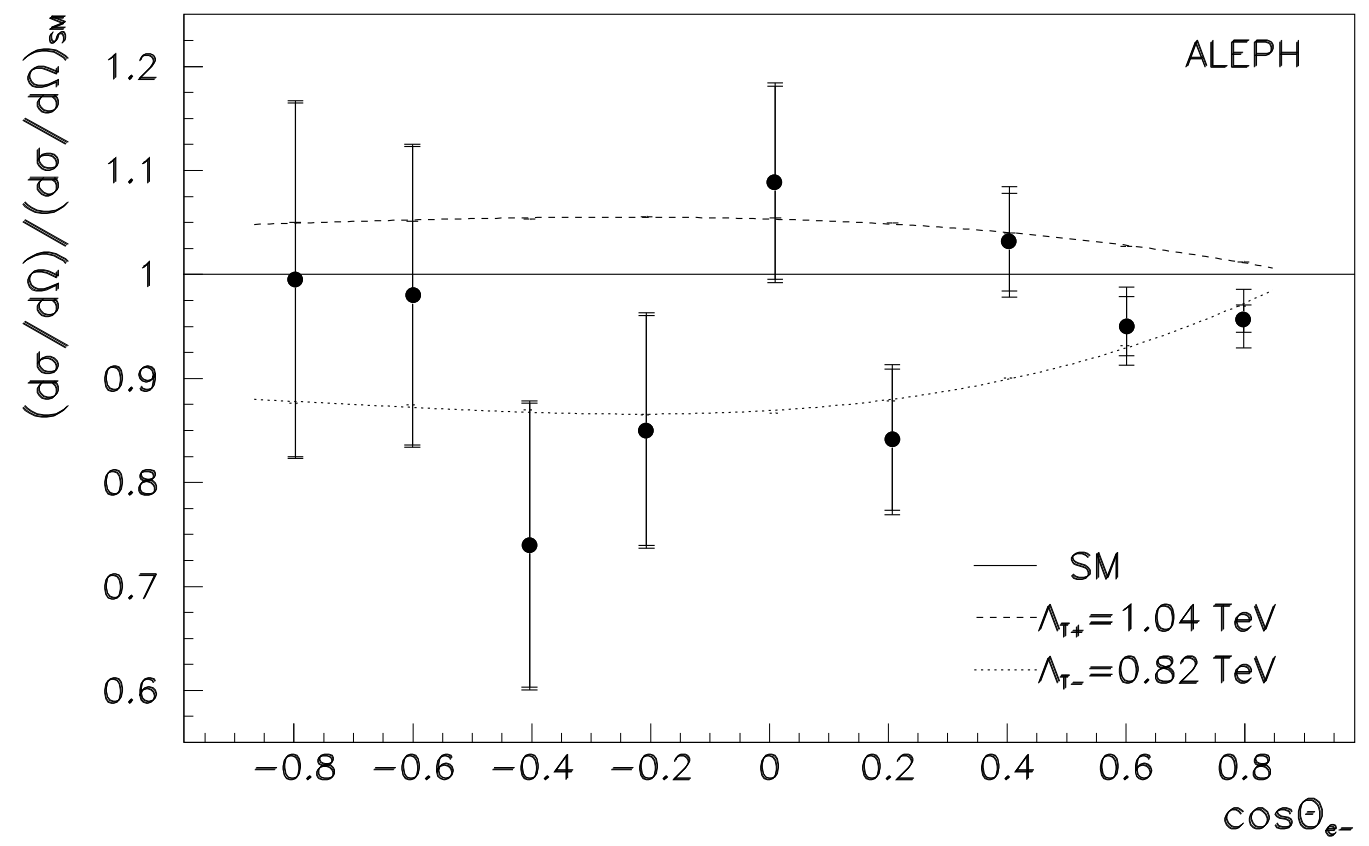

Figure 4: The ratio of the observed to the predicted differential cross section for $e^{+} e^{-} \rightarrow e^{+} e^{-}$as a function of $\cos \theta$, where $\theta$ is the $e^{-}$production angle relative to the $e^{-}$beam. Only the results from $\sqrt{s}=189 \mathrm{GeV}$ are shown. The inner (outer) error bars represent the uncertainties on the data (data + theory). The dashed and dotted lines correspond to the cross section ratios which include the effects from virtual graviton exchange with $\Lambda_{T}^{+}\left(\Lambda_{T}^{-}\right)$set equal to its $95 \%$ C.L. lower limit of 1.04 (0.82) $\mathrm{TeV}$.

The measured $q \bar{q}$ and $b \bar{b}$ cross sections give little sensitivity as explained in Section 5.1. The limits derived with these channels come primarily from the jet-charge forward-backward asymmetry measurements, but these have rather poor precision. 


\section{Combined Results on $\Lambda_{T}$ :}

As described in sections 4 and 5 above, independent measurements have been made of $\left(1 / \Lambda_{T}\right)^{4}$ in the reactions $e^{+} e^{-} \rightarrow \gamma \gamma$ and $e^{+} e^{-} \rightarrow \mathrm{f} \bar{f}$, respectively. Adding together the log-likelihood functions, a combined measurement can be derived. The result is:

$$
\left(1 / \Lambda_{T}\right)^{4}=-1.08_{-0.56}^{+0.58} \mathrm{TeV}^{-4}
$$

There is no statistically significant evidence for a value of $\left(1 / \Lambda_{T}\right)^{4}$ which differs from zero. Therefore, $95 \%$ confidence level lower limits on $\Lambda_{T}^{+}$and $\Lambda_{T}^{-}$will be given:

$$
\Lambda_{T}^{+}>1.12 \mathrm{TeV}
$$

and

$$
\Lambda_{T}^{-}>0.84 \mathrm{TeV}
$$

\section{Conclusions}

A variety of searches for effects due to extra spatial dimensions in the context of $\mathrm{TeV}$ scale quantum gravity has been performed in ALEPH at LEP-1 and LEP-2. No significant effects are found either in direct production of gravitons (including scalar gravitons) or as modifications to Standard Model processes due to virtual graviton exchange.

A measurement of the process $e^{+} e^{-} \rightarrow \gamma \not E$ at center of mass energies in the range 161 to 189 $\mathrm{GeV}$ is in agreement with Standard Model expectations. No significant signal for graviton $(\mathrm{G})$ production in the process $e^{+} e^{-} \rightarrow \gamma \mathrm{G}$ is observed. Lower limits, at the $95 \%$ confidence level, on the mass scale $M_{D}$ are established as $0.99 \mathrm{TeV}$ to $0.49 \mathrm{TeV}$, for two to six extra spatial dimensions. The corresponding upper limits on the size $R$ of the extra spatial dimensions range from $0.49 \mathrm{~mm}$ down to $69 \mathrm{fm}$.

A search with LEP-1 data for scalar graviton $\left(\mathrm{G}_{0}\right)$ production in $\mathrm{Z} \rightarrow \mathrm{f} \overline{\mathrm{f}} \mathrm{G}_{0}$ finds no significant signal. Lower limits on $M_{D}$ are found to be in the range 0.35 to $0.12 \mathrm{TeV}$.

Measurements of the differential cross sections for $e^{+} e^{-} \rightarrow \gamma \gamma$ and $e^{+} e^{-} \rightarrow \mathrm{f} \bar{f}$ have been made at LEP-2 at center of mass energies up to $189 \mathrm{GeV}$. There is no evidence for a deviation from the Standard Model due to the exchange of virtual gravitons. Lower limits at the $95 \%$ confidence level of $1.12 \mathrm{TeV}$ and $0.84 \mathrm{TeV}$ are thereby established on the respective ultraviolet cutoff parameters $\Lambda_{T}^{+}$and $\Lambda_{T}^{-}$.

\section{Acknowledgements}

We would like to thank Savas Dimopoulos, Gian Giudice, and Jim Wells for helpful discussions.

\section{References}

[1] N. Arkani-Hamed, S. Dimopoulos and G. Dvali, "The Hierarchy Problem and New Dimensions at a Millimeter", Phys. Lett. B429 (1998) 263. 
[2] N. Arkani-Hamed, S. Dimopoulos and G. Dvali, "Phenomenology, Astrophysics and Cosmology of Theories with Sub-Millimeter Dimensions and TeV Scale Quantum Gravity", Phys. Rev. D59 (1999) 86004.

[3] J. L. Hewett, "Indirect Collider Signals for Extra Dimensions", SLAC-PUB-8001 (November, 1998); hep-ph/9811356.

[4] G. F. Giudice, R. Rattazzi, and J. Wells, "Quantum Gravity and Extra Dimensions at HighEnergy Colliders", Nucl. Phys. B544 (1999) 3 and errata (to be published); hep-ph/9811291.

[5] ALEPH Collaboration, "Searches for Supersymmetry in the Photon(s) plus Missing Energy Channels at $\sqrt{s}=161$ GeV and 172 GeV", Phys. Lett. B420 (1998) 127.

[6] ALEPH Collaboration, "Single and Multi-photon Production in $e^{+} e^{-}$Collisions at a Centreof-mass Energy of 183 GeV", Phys. Lett. B427 (1998) 201.

[7] ALEPH Collaboration, "Single and Multi-photon Production in $e^{+} e^{-}$Collisions at a Centreof-mass Energy of 189 GeV", ALEPH CONF/99-025 (June, 1999), contribution to the 1999 summer conferences.

[8] C. Balazs, H.-J. He, W. W. Repko, C.-P. Yuan and D. A. Dicus, "Collider Tests of Compact Space Dimensions Using Weak Gauge Bosons", MSUHEP-90105 (April, 1999); hep$\mathrm{ph} / 9904220$.

[9] ALEPH Collaboration, "Search for Invisible Higgs Boson Decays in $e^{+} e^{-}$Collisions at Centre-of-mass Energies up to 184 GeV", Phys. Lett. B384 (1999) 301.

[10] ALEPH Collaboration, "Study of Fermion Pair Production in $\mathrm{e}^{+} \mathrm{e}^{-}$Collisions at 130183 GeV", CERN-EP/99-042, (submitted to Euro. Phys. J. C).

[11] ALEPH Collaboration, "Fermion Pair Production in $\mathrm{e}^{+} \mathrm{e}^{-}$Collisions at 189 GeV and Limits on Physics Beyond the Standard Model", ALEPH CONF/99-013.

[12] S. Jadach, W. Placzek and B.F.L. Ward, "BHWIDE 1.00: O(alpha) YFS exponentiated Monte Carlo for Bhabha Scattering at wide angles for LEP-1/SLC and LEP-2", Phys. Lett. B390 (1997) 298.

[13] D. Bardin et al., "Zfitter: An Analytical Program for Fermion Pair Production in $e^{+} e^{-}$ Annihilation", CERN-TH 6443/92.

[14] M. Martinez et al., "Model independent fitting to the Z line shape", Z. Phys. C49 (1991) 645. 